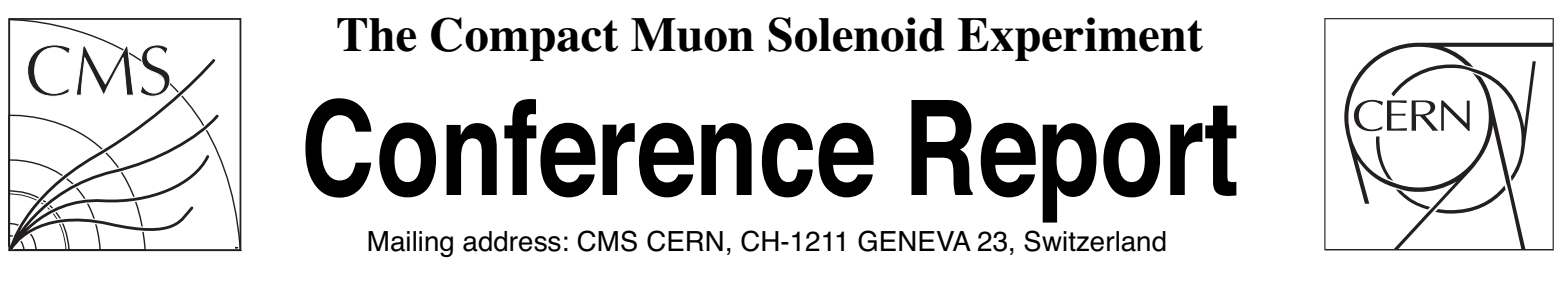

21 August 2009 (v3, 12 September 2009)

\title{
New physics at ATLAS and CMS with the first data
}

Taylan Yetkin for the CMS Collaboration

\begin{abstract}
A new era in particle physics is about to begin. The long-awaited $p p$ collisions at LHC will take place later this year. The two general purpose experiments, ATLAS and CMS, will collect first physics data in collisions of $5 \mathrm{TeV}$ proton beams, by which $\sim 200$ total data is expected to be delivered by the end of the first 2009-2010 run. The ATLAS and CMS detectors are designed as discovery machines for the missing piece in Standard Model -the Higgs boson- and for physics beyond the Standard Model. Both experiments have conducted detailed analyses to show the discovery potential for new physics, including SUSY, extra dimensions, and new heavy resonances. In this note, after giving a brief overview of the status of the two experiments, I will present results from ATLAS and CMS studies for the expected new physics reach with early LHC data.
\end{abstract}

Presented at BSM-LHC09: Beyond the Standard Model Physics and LHC Signatures (BSM-LHC) 


\title{
New Physics at ATLAS and CMS Experiments with the First Data*
}

\author{
T. Yetkin ${ }^{\mathrm{a}}$

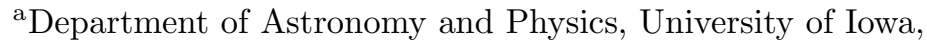 \\ Iowa City, IA 52242-1479, USA \\ (For ATLAS and CMS Collaborations)
}

\begin{abstract}
A new era in particle physics is about to begin. The long-awaited $p p$ collisions at LHC will take place later this year. The two general purpose experiments, ATLAS and CMS, will collect first physics data in collisions of 5 TeV proton beams, by which $\sim 200 \mathrm{pb}^{-1}$ total data is expected to be delivered by the end of the first 2009-2010 run. The ATLAS and CMS detectors are designed as discovery machines for the missing piece in Standard Model-the Higgs boson- and for physics beyond the Standard Model. Both experiments have conducted detailed analyses to show the discovery potential for new physics, including SUSY, extra dimensions, and new heavy resonances. In this note, after giving a brief overview of the status of the two experiments, I will present results from ATLAS and CMS studies for the expected new physics reach with early LHC data.
\end{abstract}

\section{Introduction}

The Large Hadron Collider (LHC) [1] project at CERN Laboratory (the European laboratory for particle physics near Geneva, Switzerland) is expected to be operational starting from October in 2009. When the collider reaches to its design capabilities it will collide protons at center of mass (c.o.m.) energy of $14 \mathrm{TeV}$, and with these collisions new frontiers will be opened in particle physics to understand the very fundamentals of particles and forces. The collisions at the first phase of the LHC program, however, will take place at a lower of c.o.m. energy, and it is expected to be at $10 \mathrm{TeV}^{2}$.

The LHC accelerator ring is equipped, along with two special purpose detectors (LHCb [3] and ALICE [4]), with two general purpose detectors: ATLAS [5] and CMS [6]. These state-of-the-art detectors are built to look for the Higgs particle, which is introduced to provide electroweak symmetry breaking $[7,8]$, but both detectors are also perfectly capable of providing insights for

\footnotetext{
${ }^{*}$ Presented at BSM LHC Conference, Boston, MA, 2-4 June, 2009.

${ }^{2}$ After the workshop, a new plan was announced by LHC project. According to the plan the collisions will start at $\sqrt{s}=7$ and then be increased to $\sqrt{s}=10$. Please see the link given in Ref. [2] for recent updates.
}

new physics (the term "new physics" refers to physics models that have been built to explain the shortcomings of the Standard Model, and it is often used interchangeably with Beyond Standard Model (BSM)).

The ATLAS and CMS detectors, despite their differences in size and technology they use, share same design idea: they are built to have multiple concentric layers surrounding the beam pipe, and each of these performs a different measurement of particle property. In the end, there will be position, four-momentum, and time information for the particles that interacted with the detectors as a whole system. The potential of the detectors for new physics was discussed in detail by both collaborations in Refs. $[9,10]$. Even at lower c.o.m. collision energies, the ATLAS and CMS detectors are expected to probe sensitive mass regions near where new physics is conjectured. In this note, I will shortly summarize the BSM studies performed for $\sqrt{s}=14 \mathrm{TeV} p p$ collisions, especially for exotic and supersymmetry (SUSY) searches, for ATLAS and CMS collaborations. However, recent analysis results and new analysis studies for lower c.o.m. energies can be found in Refs. [11,12]. 


\section{Status of the Detectors and LHC Sched- ule}

After the installation, the ATLAS and CMS detectors had undergone serious commissioning activities in their caverns. The final phases of the commissioning have been done by collecting cosmic muon data where the data were used for commissioning of sub-detectors and detectors as a whole system, and, for alignment and calibration of the systems. Both detectors are being kept online with various specific tasks provided by the sub-detector groups by taking cosmic muon runs or localized runs prior to first collisions.

On September $10^{t h}$, 2008, $450 \mathrm{GeV}$ proton beams were successfully circulated around the LHC accelerator ring and they were dumped to tertiary collimators which are $\sim 150 \mathrm{~m}$ away from the detectors. Both detectors saw large amount of particle flux as the secondary particles from the beam dump, and the detectors registered large signals in the detectors $(\mathcal{O}(1000) \mathrm{TeV}$ of energies). Apart from being a symbolic startup for LHC and a success for accelerator operations, these events served to both detectors by producing valuable information, such as correlation between sub-detector readouts and tests of the signal timings. The unfortunate incident occurred on September $19^{t h}$, which was due to a bad welding splice used between two magnets, caused a delay in LHC start-up plans. Current expectations are such that the $p p$ collisions in LHC will start in late October this year at lower c.o.m. energy. After the commissioning runs, physics runs will start first with low number of bunches and protons per bunch, then it will continue with increased bunch numbers. Also, it is expected to have luminosity increase (up to $1.1 \times 10^{32}$ ) throughout the first run. In total, both experiments expect to collect around $200-300 \mathrm{pb}^{-1}$ of data. The experiments will exploit the first tens of $\mathrm{pb}^{-1}$ of data for re-discovery of the Standard Model, by which detectors will be more understood and ready for discovery studies. With the additional data at the order of $100 \mathrm{pb}^{-1}$, full searches for new physics will start.

\section{Beyond the Standard Model and the LHC}

The ATLAS and CMS detectors are designed for the discovery of the Higgs boson, the particle which is believed to be responsible from the electroweak symmetry breaking. Nevertheless, there are other aspects in today's particle physics which do not find themselves in the picture that Standard Model provides (interested reader can find very detailed discussions in Refs. [13-17]). Moreover, while the boundary between particle physics and cosmology becomes more and more blurry, certain observations made in cosmology demand explanations that the Standard Model cannot give, such as the origin of dark matter and dark energy. The LHC collisions will allow scientists to probe smaller distances at very high event rates where new symmetries or particles will be found at the detectors, and with these findings some of the basic questions in particle physics and cosmology Beyond the Standard Model (BSM) will be revealed.

The studies of BSM theories in ATLAS and CMS experiments are generally grouped into two categories; supersymmetry (SUSY) and exotic searches. In the former category, the evidence for SUSY is searched by forming analysis which are sensitive to large missing transverse energy $\left(E_{\mathrm{T}}^{\mathrm{miss}}\right)$, high energy multi-jets and multiple isolated leptons. In the latter category, different types of analysis are established depending on the model under investigation. In the following subsections several analysis studies will be summarized from each category.

\subsection{Exotic Physics Searches}

One of the puzzles of today's physics is the apparent difference between mass scales associated with the forces of the nature. A novel way of solving this hierarchy problem was introduced with the idea of extra dimensions. There are three main models in the literature: ADD model (named after Arkani-Hamed, Dimopoulos, and Dvali who proposed it), Randall-Sundrum model, and UED (for universal extra dimensions). These models provide new signatures to look for in collider experiments such as black holes, mass reso- 
nances, mono-jets with $E_{\mathrm{T}}^{\text {miss }}$.

In the ADD model [18-20], in which $d$ extra flat spacelike dimensions are added, the particles of Standard Model are confined to 3-dimensional brane (our space), but gravity propagates in all dimensions. In this model, virtual graviton exchange, direct graviton production, and black hole formations are allowed. As an example, a process is considered by the CMS experiment when a graviton is produced along with a quark or gluon [21]. Since the graviton behaves as stable and non-interacting massive particle in this model, it leaves the detector undetected. The signature for this process, therefore, is large $E_{\mathrm{T}}^{\text {miss }}$ and a single jet with high $p_{\mathrm{T}}$. In this model, the number of extra dimensions and Planck Mass scale are the parameters that can be searched experimentally. By requiring one high $p_{\mathrm{T}}$ jet and large $E_{\mathrm{T}}^{\text {miss }}$ that are in opposite directions in the transverse plane, we can select such events from the LHC collisions. After applying selection cuts and performing background analysis (by stating that a very good understanding of $\mathrm{W} / \mathrm{Z}+$ jets backgrounds is crucial), CMS experiment found that with the $100 \mathrm{pb}^{-1}$ of data with this model, Planck Mass scale up to $5 \mathrm{TeV}$ with two or four extra dimensions can be searched. Also, it was shown that discovery with $5 \sigma$ significance can be made with similar amount of data (Fig. 1).
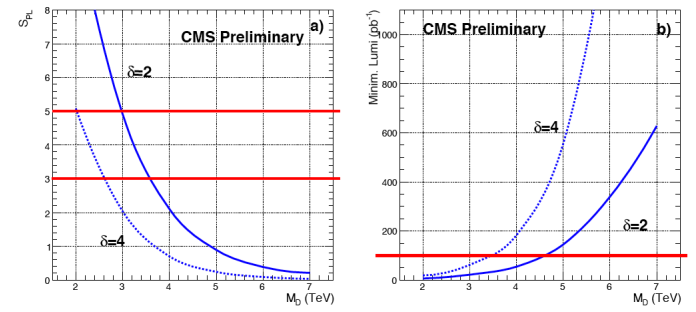

Figure 1. Significance of the discovery potential for different $M_{D}$ (where $M_{D}$ is the fundamental mass scale) and number of extra dimensions (left), and required minimum integrated luminosity for the $95 \%$ C.L. exclusion for $M_{D}$ with different number of extra dimensions (right) for CMS experiment. From Ref. [21].
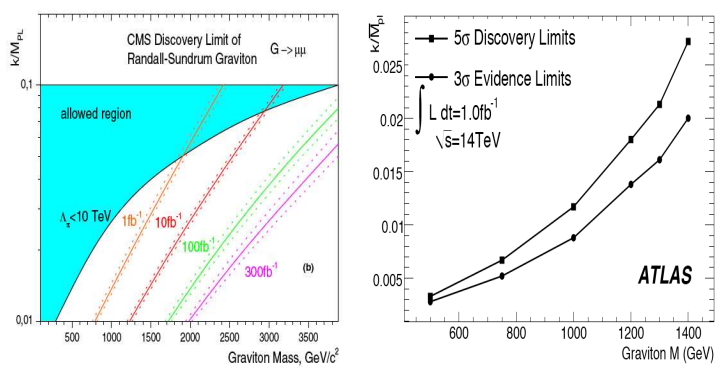

Figure 2. Left: $5 \sigma$ discovery reach for the CMS with various integrated luminosity values in coupling constant versus graviton mass (from Ref.[10]). Right: Dependence of $5 \sigma$ discovery and $3 \sigma$ evidence limits on the graviton mass and coupling constant for ATLAS (from Ref. [9])

The Randall-Sundrum [22,23] model of extra dimensions is found by solving Einstein's field equations for a metric of 5-dimensional warped (known as anti de Sitter-AdS-) spacetime. In the simplest version of this model, the particles of Standard Model are confined to $\mathrm{TeV}$ brane (which is our universe), and the gravity extends to the fifth dimension. Also, the graviton interacts with the particles of Standard Model on the TeV brane and decay into particles that can be detected. Two of such cases are studied by ATLAS and CMS experiments $[9,10]$ where a graviton decays into muon and electron pairs, respectively (Fig. 2). The CMS study concluded that graviton masses with low $c$, which is the dimensionless Randall-Sundrum coupling constant defined as k $/ \bar{M}_{\mathrm{Pl}}$ (where $\bar{M}_{\mathrm{Pl}}$ implies the reduced Planck Mass) will require more data to discover, but with $c=0.1$, the graviton masses up $\sim 1 \mathrm{TeV}$ will be observable with $1 \mathrm{fb}^{-1}$ of data. In the ATLAS study it was also shown that, with the first data, lower coupling constant regions can be explored and $5 \sigma$ discovery limits can be reached.

Another exciting outcome of extra dimension models is the possibility of creating black holes in $p p$ collisions. Extra dimensions allow stronger gravity than what we observe in our spacetime, therefore, larger event horizon radius for massive objects becomes possible. As a result, the 
impact parameter of the two colliding partons in LHC can be smaller than their horizon radius. In such processes, strong gravitational effects will dominate the other forces and black holes can be formed [24-26]. Since the black hole will evaporate very rapidly via the Hawking radiation $[27,28]$, there will be isotropic spray of particles from the black hole. Thus, the mass of the black hole can be inferred from its decay products. As a result, an experimentally sensitive parameter can be formed with the invariant mass or scalar sum of the transverse momentum of all the particles that are seen in the detector. The ATLAS and CMS experiments performed analysis with the black hole events generated in the mass range of LHC reach, where the contribution from Standard Model processes was vastly reduced by using a cut value of $2.5 \mathrm{TeV}$ on the probing parameter (Fig. 3). CMS experiment concluded that Planck Mass scale up to 2-3 $\mathrm{TeV}$ with extra dimensions up to 6 , and minimum black hole mass up to $4 \mathrm{TeV}$ can be probed with the first data $\left(<100 \mathrm{pb}^{-1}\right)$, but probing higher Planck Mass scale will require more data. ATLAS study, which differs from the CMS study by the model they used, found that black holes above a $5 \mathrm{TeV}$ production threshold can be discovered with a few $\mathrm{pb}^{-1}$ of data. With $1 \mathrm{fb}^{-1}$ of data, the black hole discovery can be made even the production thresholds was $8 \mathrm{TeV}$.

The CP violation explains the matterantimatter imbalance in the universe [29]. In the Standard Model, the chiral quark mass phase term provides a mechanism for $\mathrm{CP}$ violation but this source is too small to explain the asymmetry in the Universe. Special machines as called "B factories" have been designed to solve this mystery (Belle [30], BaBar [31]). On the other hand, the notion of fourth generation quarks is introduced to enhance $\mathrm{CP}$ phase term to solve this problem $[32,33]$. As an experimental probe, a bottom-like quark with a mass above the total mass of top quark and $W$ boson is considered by the CMS experiment, where $b^{\prime} \bar{b}^{\prime}$ is the end product [34]. Because of the mass relation chosen, the decay channel $b^{\prime} \rightarrow W t$ is expected to be dominant. When both $b^{\prime}$ quarks
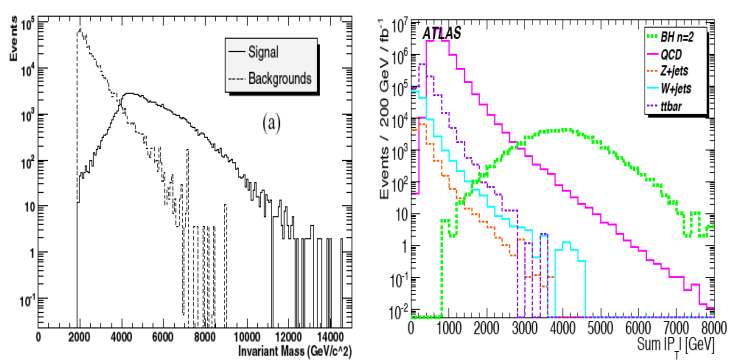

Figure 3. Left: Invariant mass of all the particles from black hole radiation for $n=3$ extra dimensions in CMS (from Ref. [10]). Right: Scalar sum of $p_{\mathrm{T}}$ of all particles from black hole radiation for $n=2$ extra dimensions in ATLAS (from Ref. [9]). The background from Standard Model falls very rapidly for black hole masses above $\sim 3 \mathrm{TeV}$.

decay into a top quark and a $W$ boson, the final state contains four $W$ bosons. The basic signature becomes $b b W W W W$ where each $W$ boson can either decay leptonically or hadronically. This leads to a distinct signature with multi-jets, multi-leptons and $E_{\mathrm{T}}^{\text {miss }}$. Especially same-sign dilepton or trilepton channels are major channels with very small background. A probing parameter, $H_{\mathrm{T}}\left(\equiv \sum p_{\mathrm{T}}(\right.$ jets $)+\sum \mathrm{p}_{\mathrm{T}}$ (leptons) $\left.+\mathrm{E}_{\mathrm{T}}^{\text {miss }}\right)$, can be used to observe signal above the background. As shown in Fig. 4, with the increased $b^{\prime}$ mass, the contribution from the Standard Model background increases, but $5 \sigma$ discovery remains still achievable with first data. This channel provides a unique way to test fourth generation quark model.

As an another example of exotic searches a new type of particle, heavy, stable and charged one (hence the name HSCP), is predicted in some BSM theories [35]. HSCPs are expected to be produced in pair production processes or in the end of decay chain of BSM particles. These new particles can hadronize into metastable states in the detectors, and then decay into Standard Model particles or into new stable particles. As shown by the CMS study [36], their existence can be traced with the timing information from the tracker and drift chambers or with the ionization 

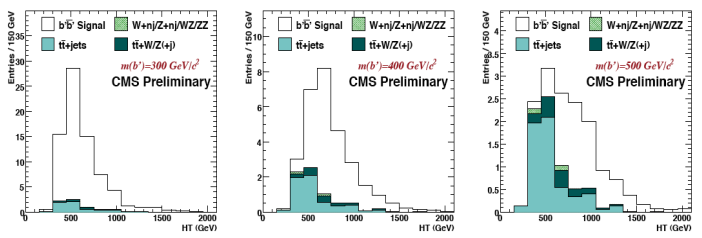

Figure 4. $H_{\mathrm{T}}$ distribution for various values of $b^{\prime}$ quark mass ( $300 \mathrm{GeV} / \mathrm{c}^{2}$ (left), $400 \mathrm{GeV} / \mathrm{c}^{2}$ (middle), $500 \mathrm{GeV} / \mathrm{c}^{2}$ (right)) where same-sign dilepton and trilepton channels are added together. From Ref. [34].

loss (Fig. 5). Because of the delay in HSCP's decay time, the contribution from the Standard Model particles can easily be removed by applying a cut on both axes. Furthermore, special triggers that trigger on such events in between collisions can be implemented to eliminate the Standard Model background. In Fig. 6 the required integrated luminosity for $5 \sigma$ discovery reach is shown for different HSCPs. It was found that, stable gluino and stau with the masses 500-600 $\mathrm{GeV}$ range can be discovered with $100 \mathrm{pb}^{-1}$ of data, but HSCP from different models or heavier ones will require more data to claim for discovery.
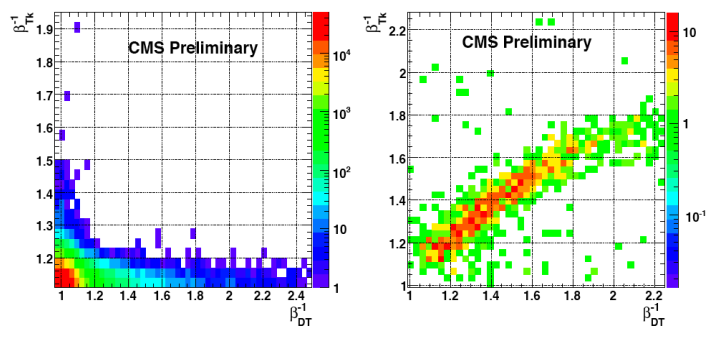

Figure 5. Distribution of $\beta_{\mathrm{Tk}}^{-1}$ (ionization loss measurement from the tracker) versus $\beta_{\mathrm{DT}}^{-1}$ (ionization loss measurement from the drift tubes) for background (left) and for the signal sample (right). Here $\beta^{-1} \sim \sqrt{d E / d x}$ ). From Ref. [36].

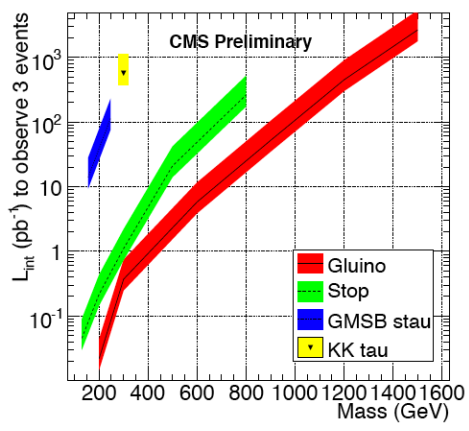

Figure 6. Required integrated luminosity for observing 3 excess events versus HSCP mass in four different models. From Ref. [36].

\subsection{Supersymmetry Searches}

In the course of the development of string theory and approaches for grand unified theories a new symmetry, supersymmetry, is emerged (see Refs. [37-42] for very comprehensive introduction and applications in particle physics). Supersymmetry (SUSY) predicts that every particle in Standard Model has a partner with a spin by $1 / 2$ unit difference. Therefore, fermions and bosons are related, i.e., one can be turned into other under SUSY operations. The SUSY partners of particles (sparticles) have not been seen in cosmology observations or in collision experiments, therefore SUSY must be broken, but the exact SUSY breaking mechanism is unknown. There are many SUSY models, with their free parameters, that provides breaking mechanism. These models lead to different signatures in the detectors. Experimentally it is challenging to perform analysis for every model that exists. Thus, a generalized version of SUSY (mSUGRA), which is derived from supergravity with minimal superpotential, is mostly used in experimental search. In the mSUGRA model gaugino and scalar masses, trilinear coupling constant are universal at a high mass scale, and there are only five parameters that determine the mass of the sparticles. Also, since it gives a candidate particle for the dark matter, the R-parity conserved SUSY model is one of the most studied model in collider experiments. As a result of R-parity conservation, sparticles are pair produced and the lightest spar- 
ticle, LSP, is stable. The LSP weakly interacts with the matter and leaves no trace in the detector, therefore it appears as missing energy in the measurements. The cascade decays of gluinos and squarks lead to multi-jets and multi-lepton signals in the detector. Furthermore, as another generalization and to develop analysis methods, both ATLAS and CMS experiments have built benchmark points in the so called mSUGRA plane (common scalar mass $m_{0}$ vs. common gaugino mass $m_{1 / 2}$ ), where some of the five parameters are varied freely. Each point in this plane represent different event kinematics and physics objects. By this way, analysis studies are developed to cover all aspects of expected SUSY signals.

If the $E_{\mathrm{T}}^{\mathrm{miss}}$ is well understood during the early data taking, SUSY discovery with large $E_{\mathrm{T}}^{\text {miss }}$ and high multi-jets is very plausible at the beginning of LHC collisions. Both experiments have demonstrated detailed analysis for this channel. One of the parameters that will show evidence for the SUSY is the effective mass, where $p_{T}$ of the jets is added to the $E_{\mathrm{T}}^{\text {miss }}$ scalarly. As noted by the ATLAS and CMS experiments, the dominant backgrounds for this channel are $\mathrm{QCD}, t \bar{t}$, $W+$ jets and $Z$ to invisible decays (Fig. 7). The studies showed that excess events above $5 \sigma$ reach will be observable with $1 \mathrm{fb}^{-1}$ data if mass of the SUSY particles are $\mathcal{O}(1 \mathrm{TeV})$.

In addition to large $E_{\mathrm{T}}^{\text {miss }}$ and multiple high $p_{\mathrm{T}}$ jets, the use of isolated leptons in SUSY analysis will yield better results. One of the benefits of having isolated lepton(s) in the analysis is the increased QCD background rejection, as shown in Fig. 8(left). In the analysis performed by ATLAS experiment, the event selections were kept as same as multi-jet $+E_{\mathrm{T}}^{\text {miss }}$ analysis (0 lepton mode), but one isolated lepton was added. The observed signal yield above background was found as higher in this channel. Fig.8(right) shows another example of lepton analysis from CMS. In cascade decays of sparticles, neutralinos can give two isolated leptons as $\tilde{\chi}_{2}^{0} \rightarrow l l \tilde{\chi}_{1}^{0}$. Because of the kinematical constrains on the threebody decay, mass edges can be observed in dilepton analysis. This type of analyses are other way of SUSY discovery but they only yield better re-
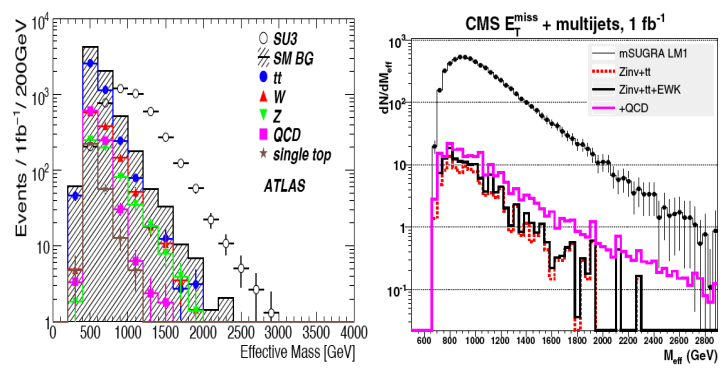

Figure 7. Effective mass $\left(M_{\text {eff }}\right)$ distribution for SUSY signal and backgrounds in multijets $+E_{\mathrm{T}}^{\mathrm{miss}}$ channel in ATLAS (left) and CMS (right). The contribution from the background is expected to be smaller with the increased $M_{\text {eff }}$, which is a parameter closely related to the SUSY mass scale. From Refs. $[9,10]$.

sults with more data.

Recently, another perspective have been brought up by Randall and Tucker-Smith [44] for SUSY searches in which they invented a new variable, $\alpha$. It is defined as the ratio of the $p_{\mathrm{T}}$ of the second leading jet and the invariant mass formed from the two leading jets, $p_{T}^{j 2} / m_{j 1, j 2}$. Then, by CMS, due to practical purposes, the $\alpha$ variable was re-defined as $\alpha_{\mathrm{T}}=p_{\mathrm{T}}^{j 2} / m_{\mathrm{T}}(j 1, j 2)$ in transverse plane. When there is no heavy invisible particles escaping from the detector and the jets are back-to-back, and the $\alpha_{\mathrm{T}}$ distribution can have a value at most 0.5. However, $\alpha_{\mathrm{T}}$ becomes larger when jets are not back-to-back and in SUSY, events are expected to be seen in the regions where $\alpha_{\mathrm{T}}$ is greater than 0.5. CMS experiment has studied dijet events for SUSY discovery and found that $\alpha_{\mathrm{T}}$ can be a strong discriminator against QCD background as shown in Fig. 9. One of the merits of this study was the use of data driven methods for background estimation for $\mathrm{QCD}$ and $Z \rightarrow \nu \nu$ components. With this study, where $E_{\mathrm{T}}^{\text {miss }}$ information was not used, it was shown that $5 \sigma$ discovery is achievable with $100 \mathrm{pb}^{-1}$ data.

As mentioned before, QCD is one of the the major backgrounds in SUSY searches due to large 

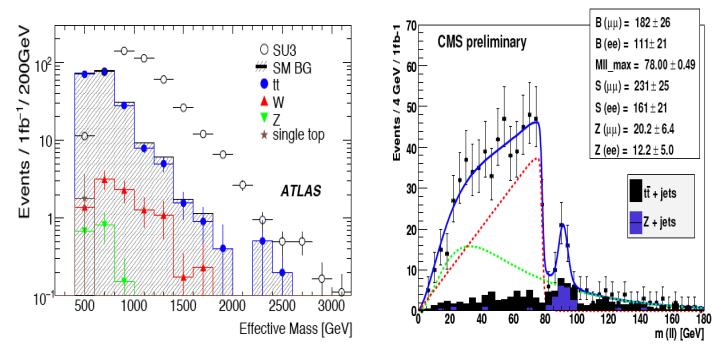

Figure 8. Left: Effective mass $\left(M_{\text {eff }}\right)$ distribution for a SUSY benchmark point and expected backgrounds in multi-jets $+E_{\mathrm{T}}^{\text {miss }}+$ isolated lepton channel in ATLAS (from Ref. [9]). Right: Dilepton mass distribution for SUSY and various important background components in CMS (from Ref. [43]).

$E_{\mathrm{T}}^{\text {miss }}$ events produced with a high rate in these processes. Especially multi-jet $+E_{\mathrm{T}}^{\text {miss }}$ analysis will be dominated by such events. Some fraction of the large $E_{\mathrm{T}}^{\text {miss }}$ in QCD processes is caused by the $b \bar{b}$ and $c \bar{c}$ production, but significant contribution comes from jet mismeasurements and calorimeter effects. When a jet is mismeasured, the $E_{\mathrm{T}}^{\mathrm{miss}}$ vector tends to be aligned with the jet $p_{\mathrm{T}}$ vector, i.e. $E_{\mathrm{T}}^{\text {miss }}$ vector becomes closer to jet $p_{\mathrm{T}}$ vector in $\phi$. Therefore, two angle variables between the jets $p_{\mathrm{T}}$ and $E_{\mathrm{T}}^{\mathrm{miss}}$ vectors are defined as $\left|\Delta \phi\left(j 1, E_{\mathrm{T}}^{\text {miss }}\right)\right|,\left|\Delta \phi\left(j 2, E_{\mathrm{T}}^{\text {miss }}\right)\right|$ and their scatter plot is used to reject events which have large $E_{\mathrm{T}}^{\text {miss }}$ in QCD because of these mismeasurements $[46,47]$. These variables were also used by ATLAS and CMS detectors for the QCD events that are expected from LHC collisions, and it was shown that this rejection criteria is very powerful for QCD events while retaining almost all of the SUSY events $[9,10]$.

However, the topological variables $\left(\left|\Delta \phi\left(j 1, E_{\mathrm{T}}^{\text {miss }}\right)\right|, \quad\left|\Delta \phi\left(j 2, E_{\mathrm{T}}^{\text {miss }}\right)\right|\right)$ are not expected to remove all the large $E_{\mathrm{T}}^{\text {miss }}$ events from QCD background. Therefore, other methods are needed to estimate its contribution in the signal region. Two methods, namely matrix (or ABCD) [45] method and $E_{\mathrm{T}}^{\text {miss }}$ projection method [9] were used to count QCD background events. In the former method, if two variables

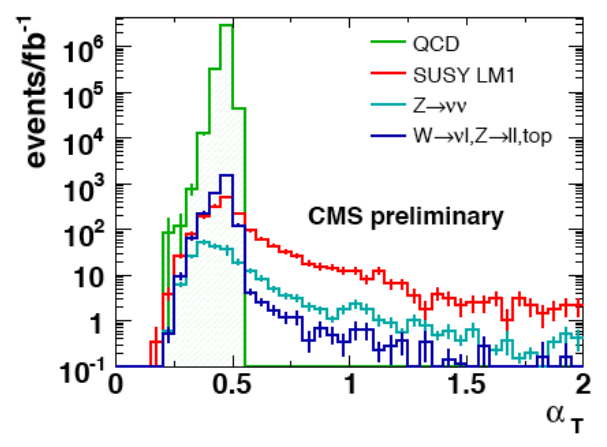

Figure 9. Distribution of $\alpha_{\mathrm{T}}$ for a SUSY benchmark point and various backgrounds in CMS. From Ref. [45].

are found to be uncorrelated for background, and two signal depleted regions and one signal rich region can be defined by plotting these variables against each other, then the number of background events in the signal region can be estimated with the simple extrapolation formula. This method was used in one of the SUSY analysis to estimate number of background events (Fig. 10). In the latter method, the $E_{\mathrm{T}}^{\text {miss }}$ was estimated with the combination of multiple steps by using jet response functions that represent Gaussian and non-Gaussian parts of the response function. The Gaussian part was found from $\gamma$-jet events and non-Gaussian part was found from the QCD multi-jet events where $E_{\mathrm{T}}^{\mathrm{miss}}$ is parallel to the one of the three leading jets, and jets are selected to have large angular separation to give symmetric jet (also referred as "mercedes type") event topology. Then, jets in low $E_{\mathrm{T}}^{\mathrm{miss}}$ data are smeared with these smearing functions to estimate the detector effects. After the smearing, the $E_{\mathrm{T}}^{\mathrm{miss}}$ is recalculated in event-by-event basis as shown in Fig. 11. Both methods give results which are comparable with Monte Carlo truth information. Nevertheless, QCD background estimation will be very crucial for SUSY studies and such different methods will be used in conjunction for optimizations and vigorous estimates.

$Z \rightarrow \nu \nu$ is an another major background for 

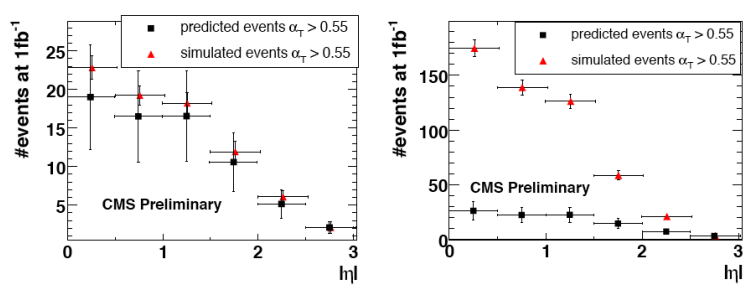

Figure 10. Left: Estimation of background events as compared with predicted events in SUSY dijet analysis. Right: Estimation of background events when contaminated with signal (black squares) and total number of observed events when the signal is present (red triangles). From Ref. [45].

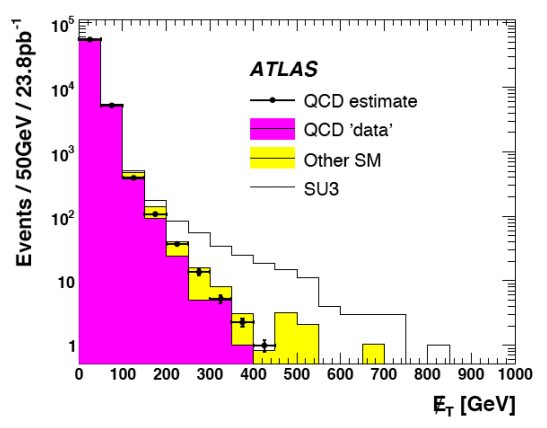

Figure 11. $E_{\mathrm{T}}^{\text {miss }}$ distribution as estimated with response function (dotted histogram). Also, $E_{\mathrm{T}}^{\text {miss }}$ distribution for QCD "data" (filled histogram with magenta color) and a SUSY benchmark point (filled histogram with yellow color). From Ref. [9].

SUSY searches because of the $E_{\mathrm{T}}^{\mathrm{miss}}$ contribution to the background events. Traditionally its contribution is estimated by using a "candle" method. First, the events that have dilepton mass in the range of $Z$ mass is found, then the leptons are treated as neutrinos in the events, i.e., after taking into account the branching ratios, the $p_{\mathrm{T}}$ of the leptons is treated as $E_{\mathrm{T}}^{\text {miss }}$. Since $B R(Z \rightarrow l l) / B R(Z \rightarrow \nu \nu)$ is small and statistical uncertainties will be large with the first data, this method will be difficult to be used in initial analysis. ATLAS and CMS experiments proposed alternative approaches to overcome this is-

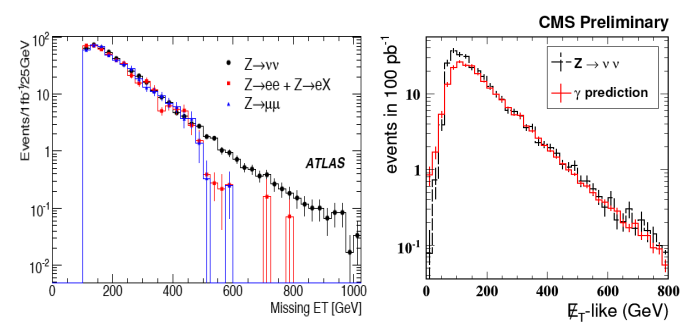

Figure 12. Left: $E_{\mathrm{T}}^{\text {miss }}$ from $Z \rightarrow \nu \nu$ and its estimation from $Z \rightarrow e e$ with relaxed candle method. Also $E_{\mathrm{T}}^{\text {miss }}$ estimation from $Z \rightarrow \mu \mu$ is shown (from Ref. [9]). Right: $E_{\mathrm{T}}^{\text {miss }}$ from $Z \rightarrow \nu \nu$ and its estimation from $\gamma$-jet events (from Ref. [48]).

sue $[9,48]$. In the method of ATLAS ("relaxed candle method"), more electron-like objects were allowed to enter $Z$ mass peak window to increase number of events for the $E_{\mathrm{T}}^{\mathrm{miss}}$ prediction. The decision on the usage of electron-like objects were made with tag and probe method, where one isolated electron is found in the event. The $E_{\mathrm{T}}^{\text {miss }}$ from $Z \rightarrow l l$ events is plotted as in the candle method. The result is shown in Fig. 12(left). The problem of low number of events in the estimated tail is solved by applying a fit to the estimated $E_{\mathrm{T}}^{\text {miss }}$ shape. The method CMS employed used the $\gamma$ or $W$-jets events to estimate $E_{\mathrm{T}}^{\text {miss }}$ expected in $Z \rightarrow \nu \nu$ events. In this approach $p_{\mathrm{T}}$ of the $\gamma$ or $W$ is treated as $E_{\mathrm{T}}^{\text {miss }}$ after taking into account the $\gamma$ or $\mu$ isolation efficiencies in branching ratio calculations for normalization procedure. The result is shown in Fig. 12(right) for the $\gamma$-jets events. Although at low $E_{\mathrm{T}}^{\text {miss }}$ there is lesser agreement between the estimation and the $E_{\mathrm{T}}^{\text {miss }}$ from $Z$ (which is where the mass of the $Z$ plays role), the $E_{\mathrm{T}}^{\mathrm{miss}}$ at high values are in very good agreement. The CMS study concluded also that the $E_{\mathrm{T}}^{\text {miss }}$ estimation from $W$-jets events suffers more from the backgrounds, but two methods can be used in conjunction with each other.

After detailed analysis in selected benchmark points, both experiments performed discovery survey with fast simulations. In these analysis object selections were optimized for the type of the analysis and mSUGRA space was scanned for 

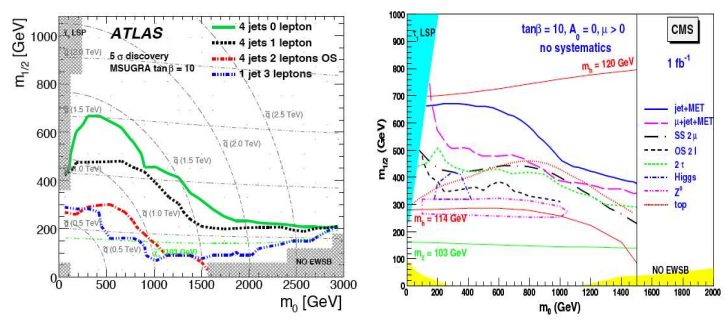

Figure 13. SUSY discovery potential with $5 \sigma$ contours given for various signatures for ATLAS [9] (left) and CMS [10](right). Both results are shown for $1 \mathrm{fb}^{-1}$.

$5 \sigma$ excess events. The ATLAS and CMS studies concluded that $5 \sigma$ reach with the multi-jets and high $E_{\mathrm{T}}^{\text {miss }}$ search channel will cover many points in mSUGRA plane(Fig. 13). Also both experiments showed the excess events above $5 \sigma$ reach will be observable with $1 \mathrm{fb}^{-1}$ of data if mass of the SUSY particles are $\mathcal{O}(1 \mathrm{TeV})$. Note also that, as found in detailed analysis, the additional objects such as leptons will help to control background better but the sensitivity to high mass SUSY will be limited in these channels with early data.

All these exotic and SUSY searches in LHC will provide new insights for physics beyond the Standard Model. A possible outcome from these searches will be a new theory where the Standard Model is embedded in it, or a completely new physics in which our understanding of particles and forces with the notion of spacetime will undergo a radical change. The data from the first collisions of LHC will allow us test these ideas only when we reach to good level of understanding of the detectors and background processes from the Standard Model as the history of experimental particle physics taught us.

\section{Conclusions}

Many new physics scenarios are already included in our efforts as the first things to look for with $100-200 \mathrm{pb}^{-1}$ data at the LHC. The analysis have shown that ATLAS and CMS detectors have the discovery potential for new physics. Also as preparation for the collisions, both ATLAS and CMS experiments were commissioned and are ready to take data. Nowadays, more efforts are being put to understand the detector in which the dissemination of the information from detector commissioning groups to analysis groups are being assured. While refining their tools for lower c.o.m. collision data (e.g., checking object selection cuts, building data driven background estimation methods, optimizing joint group efforts for successful international collaboration), both experiments are waiting for the exiting days when LHC starts colliding protons.

\section{Acknowledgments}

I thank to the organizers of BSM-LHC'09 Workshop for their kind invitation and their hospitality during the workshop. I also thank my colleagues from ATLAS and CMS experiments for all their effort that made this contribution to the workshop possible.

\section{REFERENCES}

1. The LHC Study Group, The Large Hadron Collider Conceptual Design, CERN-AC-95-05 (1995).

2. The LHC schedule can be followed from this link: http://lhc-commissioning.web. cern.ch/lhc-commissioning.

3. A. A. Augusto, et al., JINST 3 (08) (2008) S08005.

4. K. Aamodt, et al., JINST 3 (08) (2008) S08002.

5. G. Aad, et al., JINST 3 (08) (2008) S08003.

6. S. Chatrchyan, et al., JINST 3 (08) (2008) S08004.

7. J. G. Bransoni, et al., Eur. Phys. J. Direct 4 (1) (2002) 1-61. doi:10.1007/s1010502cn001.

8. A. Djouadi, Phys. Rep. 457 (1-4) (2008) 1 - 216, and references therein. doi:10.1016/j.physrep.2007.10.004.

9. G. Aad, et al., arXiV:0901.0512 (2009).

10. G. L. Bayatian, et al., J. Phys. G34 (2007) 995-1579. doi:10.1088/0954-3899/34/6/S01. 
11. For recent public results, see https: //twiki.cern.ch/twiki/bin/view/Atlas/ AtlasResults.

12. For recent public results, see https: //twiki.cern.ch/twiki/bin/view/CMS/ PhysicsResults.

13. P. Langacker, arXiv:hep-ph/0304186v1 (2003).

14. H. Murayama, arXiv:0704.2276v1 [hep-ph] (2007).

15. C. Quigg, arXiv:hep-ph/0404228 (2004).

16. J. Ellis, arXiv:hep-ph/0211168 (2002).

17. H. Baer, arXiv:0908.2785v1 [hep-ph] (2009).

18. N. Arkani-Hamed, S. Dimopoulos, G. Dvali, Phys. Let. B 429 (1998) $263 . \quad$ doi:Ball:2007zz10.1016/S03702693(98)00466-3.

19. I. Antoniadis, N. Arkani-Hamed, S. Dimopoulos, G. Dvali, Phys. Let. B 436 (3-4) (1998) 257 - 263. doi:10.1016/S0370-2693(98)008600.

20. N. Arkani-Hamed, S. Dimopoulos, G. Dvali, Phys. Rev. D 59 (8) (1999) 086004. doi:10.1103/PhysRevD.59.086004.

21. http://cms-physics.web.cern.ch/ cms-physics/public/EXO-09-013-pas . pdf.

22. L. Randall, R. Sundrum, Phys. Rev. Lett. $\quad 83 \quad$ (23) (1999) 4690-4693. doi:10.1103/PhysRevLett.83.4690.

23. L. Randall, R. Sundrum, Phys. Rev. Lett. $\quad 83 \quad$ (17) (1999) 3370-3373. doi:10.1103/PhysRevLett.83.3370.

24. S. Dimopoulos, G. Landsberg, Phys. Rev. Lett. $87 \quad$ (16) (2001) 161602. doi:10.1103/PhysRevLett.87.161602.

25. P. C. Argyres, S. Dimopoulos, J. MarchRussell, Physics Letters B 441 (1-4) (1998) 96 - 104. doi:10.1016/S0370-2693(98)01184-8.

26. S. Hossenfelder, arXiv:hep-ph/0412265v1 (2004).

27. S. W. Hawking, Commun. Math. Phys. 43 (3) 199-220. doi:10.1007/BF02345020.

28. S. B. Giddings, S. Thomas, Phys. Rev. D $65 \quad$ (5) (2002) 056010. doi:10.1103/PhysRevD.65.056010.

29. A. D. Sakharov, Pisma Zh. Eksp. Teor. Fiz. (5) (1967) 32-35, translation in JETP
Lett. 5: 24-27 (1967).

30. BELLE Collaboration, Belle Technical Design Report, KEK Report 95-1 (1995).

31. D. Boutigny, et al., BaBar Technical Design ReportSLAC-R-0457.

32. A. Arhrib, W. Hou, JHEP0607 009 (2009).

33. W.-S. Hou, arXiv:0803.1234v3 (2008).

34. http://cms-physics.web.cern.ch/ cms-physics/public/EXO-08-009-pas . pdf.

35. M. Fairbairn, et al., Phys. Rep. 438 (1) (2007) 1 - 63. doi:10.1016/j.physrep.2006.10.002.

36. http://cms-physics.web.cern.ch/ cms-physics/public/EXO-08-003-pas . pdf.

37. S. P. Martin, arXiv.org:hep-ph/9709356 (1997).

38. M. E. Peskin, arXiv.org:0801.1928 (2008).

39. K. A. Olive, arXiv.org:hep-ph/9911307 (1999).

40. M. Drees, arXiv.org:hep-ph/9611409 (1996).

41. H. E. Haber, G. L. Kane, Phys. Rep. 117 (2-4) (1985) 75 - 263. doi:10.1016/03701573(85)90051-1.

42. H. P. Nilles, Phys. Rep. 110 (1-2) (1984) 1 162. doi:10.1016/0370-1573(84)90008-5.

43. http://cms-physics.web.cern.ch/ cms-physics/public/SUS-08-001-pas . pdf.

44. L. Randall, D. Tucker-Smith, Phys. Rev. Lett. 101 (22) (2008) 1-4. doi:10.1103/PhysRevLett.101.221803.

45. http://cms-physics.web.cern.ch/ cms-physics/public/SUS-08-005-pas. pdf.

46. T. Affolder, et al., Phys. Rev. Lett. $\quad 88 \quad$ (4) (2002) 041801. doi:10.1103/PhysRevLett.88.041801.

47. V. M. Abazov, et al., Phys. Lett. B660 (2008) 449-457. arXiv:0712.3805, doi:10.1016/j.physletb.2008.01.042.

48. http://cms-physics.web.cern.ch/ cms-physics/public/SUS-08-002-pas . pdf. 